University of Nebraska - Lincoln

DigitalCommons@University of Nebraska - Lincoln

6-10-1998

\title{
Steps Toward Determination of the Size and Structure of the Broad-Line Region in Active Galactic Nuclei. XIV. Intensive Optical Spectrophotometric Observations of NGC 7469
}

\author{
S.J. Collier \\ University of St. Andrews, St. Andrews, Scotland \\ Keith Horne \\ University of St. Andrews, St. Andrews, Scotland \\ Shai Kaspi \\ Tel Aviv University, shai@wise.tau.ac.il \\ Hagai Netzer \\ Tel Aviv University, netzer@wise.tau.ac.il \\ Bradley M. Peterson \\ Ohio State University, peterson.12@osu.edu \\ See next page for additional authors \\ Follow this and additional works at: https://digitalcommons.unl.edu/physicsgaskell \\ Part of the Physics Commons
}

Collier, S.J.; Horne, Keith; Kaspi, Shai; Netzer, Hagai; Peterson, Bradley M.; Wanders, I.; Alexander, T.; Bertram, R.; Comastri, A.; Gaskell, C. Martin; Malkov, Yu. F.; Maoz, D.; Mignoli, M.; Pogge, Richard William; Pronik, V.I.; Sergeev, S.G.; Snedden, Stephanie A.; Stirpe, G.M.; Bochkarev, N.G.; Burenkov, A.N.;

Shapovalova, A.I.; and Wagner, R.M., "Steps Toward Determination of the Size and Structure of the BroadLine Region in Active Galactic Nuclei. XIV. Intensive Optical Spectrophotometric Observations of NGC 7469" (1998). Martin Gaskell Publications. 5.

https://digitalcommons.unl.edu/physicsgaskell/5

This Article is brought to you for free and open access by the Research Papers in Physics and Astronomy at DigitalCommons@University of Nebraska - Lincoln. It has been accepted for inclusion in Martin Gaskell Publications by an authorized administrator of DigitalCommons@University of Nebraska - Lincoln. 


\section{Authors}

S.J. Collier, Keith Horne, Shai Kaspi, Hagai Netzer, Bradley M. Peterson, I. Wanders, T. Alexander, R. Bertram, A. Comastri, C. Martin Gaskell, Yu. F. Malkov, D. Maoz, M. Mignoli, Richard William Pogge, V.I. Pronik, S.G. Sergeev, Stephanie A. Snedden, G.M. Stirpe, N.G. Bochkarev, A.N. Burenkov, A.I. Shapovalova, and R.M. Wagner 
ThE Astrophysical Journal, 500:162-172, 1998 June 10

(C) 1998. The American Astronomical Society. All rights reserved. Printed in U.S.A.

\title{
STEPS TOWARD DETERMINATION OF THE SIZE AND STRUCTURE OF THE BROAD-LINE REGION IN ACTIVE GALACTIC NUCLEI. XIV. INTENSIVE OPTICAL SPECTROPHOTOMETRIC OBSERVATIONS OF NGC 7469
}

\author{
S. J. Collier, ${ }^{1,2}$ Keith Horne, ${ }^{1}$ S. Kaspi,${ }^{3}$ H. Netzer, ${ }^{3}$ B. M. Peterson,,${ }^{4}$ I. Wanders,,${ }^{1,4}$ T. Alexander, ${ }^{3}$ R. Bertram, ${ }^{4,5}$ \\ A. Comastri, ${ }^{6}$ C. M. Gaskell, ${ }^{7}$ Yu. F. Malkov, ${ }^{8}$ D. Maoz, ${ }^{3}$ M. Mignoli,${ }^{6}$ R. W. Pogge, ${ }^{4}$ V. I. Pronik, ${ }^{8}$ \\ S. G. Sergeev,${ }^{8}$ S. SNedden, ${ }^{7}$ G. M. StiRPe, ${ }^{6}$ N. G. BochKarev,${ }^{9}$ A. N. BurenKov, ${ }^{10}$ \\ A. I. Shapovalova, ${ }^{10}$ AND R. M. WAGNER ${ }^{4,5}$ \\ Received 1997 September 24; accepted 1997 December 23
}

\begin{abstract}
We present results of an intensive 2 month campaign of ground-based spectrophotometric monitoring of the Seyfert 1 galaxy NGC 7469, with a temporal resolution $\lesssim 1$ day. The broad $\mathrm{H} \alpha$ and $\mathrm{H} \beta$ emission lines respond to $\sim 35 \%$ ultraviolet continuum variations with an amplitude of $\sim 10 \%$ and time delays of $5.6 \pm 1.3$ days and $5.4 \pm 0.8$ days, respectively. We interpret this as evidence of variable Balmer line gas 5-6 light days from the central source in this object, widely believed to be a supermassive black hole. The virial mass of the central source implied by line widths and time delays is $\sim 10^{6}-10^{7} M_{\odot}$. Concomitantly, we find evidence for wavelength-dependent continuum time delays: optical continuum variations lag those at $1315 \AA$ by $1.0 \pm 0.3$ days at $4865 \AA$ to $1.5 \pm 0.7$ days at $6962 \AA$. This suggests a stratified continuum reprocessing region extending several light days from the central source, possibly an accretion disk.
\end{abstract}

Subject headings: galaxies: active — galaxies: individual (NGC 7469) — galaxies: nuclei galaxies: Seyfert

\section{INTRODUCTION}

The analysis of emission lines responding to continuum variations in active galactic nuclei (AGNs) provides diagnostic information about the geometry and kinematics close to the central source (see Peterson 1993 for a review and references therein). To extract this information, we require extensive high-quality observations with temporal sampling of $\lesssim 1$ day sustained over a baseline of at least several weeks to months.

By concentrating efforts on one object each time, the AGN Watch consortium has acquired suitable monitoring data sets on NGC 5548 (Clavel et al. 1991; Peterson et al. 1991, 1992; Dietrich et al. 1993; Maoz et al. 1993; Peterson et al. 1994; Romanishin et al. 1995; Korista et al. 1995; Marshall et al. 1997), NGC 3783 (Reichert et al. 1994; Stirpe et al. 1994; Alloin et al. 1995), NGC 4151 (Crenshaw et al. 1996; Warwick et al. 1996; Kaspi et al. 1996; Edelson et al. 1996), Fairall 9 (Rodríguez-Pascual et al. 1997; Santos-Lleó et al. 1997), and 3C 390.3 (Leighly et al. 1997; Dietrich et al. 1998; O'Brien et al. 1998). These data sets generally show

\footnotetext{
${ }^{1}$ School of Physics and Astronomy, University of St. Andrews, St. Andrews, KY16 9SS Scotland.

${ }^{2}$ Visiting Observer, The Florence and George Wise Observatory, Tel Aviv University, Tel Aviv 69978, Israel.

${ }^{3}$ School of Physics and Astronomy and the Wise Observatory, The Raymond and Beverly Sackler Faculty of Exact Sciences, Tel Aviv University, Tel Aviv 69978, Israel.

${ }^{4}$ Department of Astronomy, Ohio State University, 174 West 18th Avenue, Columbus, OH 43210.

${ }^{5}$ Lowell Observatory, 1400 Mars Hill Road, Flagstaff, AZ 86001.

${ }^{6}$ Osservatorio Astronomico di Bologna, via Zamboni 33, I-40126, Bologna, Italy.

7 Behlen Observatory, University Of Nebraska, Department of Physics and Astronomy, Lincoln, NE 68588-0111.

${ }^{8}$ Crimean Astrophysical Observatory, P/O Nauchny, 334413 Crimea, Ukraine.

9 Sternberg Astronomical Institute, Moscow, Russia.

${ }^{10}$ Special Astrophysical Observatory of Russian Academy of Sciences, Nyzhnij Arkhyz, Russia.
}

UV and optical emission-line variations correlated and delayed with respect to continuum variations. The time delays for different emission lines reveal radially stratified ionization structures on scales of $\sim 2-20$ light days.

Contrary to behavior of the lines, the continuum from 1350 to $5200 \AA$ varies synchronously to within typical measurement uncertainties of \pm 2 days. At first sight, this disfavors viscous thin accretion disks as the source of the continuum variations, since mechanisms thought to produce continuum variations, e.g., disk instabilities, predict time delays between the different continuum wavelength regions of the order of hundreds of days, which are not observed. Disk models may be rescued, however, by invoking reprocessing of X-rays to coordinate continuum variations at different radii in the disk (Krolik et al. 1991).

A 2 month campaign of continuous multiwavelength monitoring was undertaken during the final episode of observations with the IUE satellite (Wanders et al. 1997), in parallel with observations by the Hubble Space Telescope (HST; Welsh et al. 1998; Kriss et al. 1998), the Rossi X-Ray Timing Explorer (RXTE; Nandra et al. 1998), and groundbased telescopes (this paper). A gyro failure on the IUE satellite in 1996 March, just two months before the start of the originally proposed campaign on Mrk 335, forced a last-minute target change. NGC 7469 was selected as a reliably variable Seyfert 1 galaxy that satisfied the constraints the gyro failure imposed. NGC 7469 is an SBa Seyfert 1 galaxy $(z=0.0164)$ that is gravitationally interacting with an irregular companion galaxy, IC 5283, approximately $80^{\prime \prime}$ away (Salamanca et al. 1995, and references therein).

The IUE monitoring campaign on NGC 7469 revealed wavelength-dependent time delays between the continuum wave bands 1315-1825 $\AA$ (Wanders et al. 1997). The continuum variations at $1825 \AA$ were observed to lag those at $1315 \AA$ by about $0.3 \pm 0.1$ days. In this paper we report results of simultaneous optical spectrophotometric monitoring of NGC 7469, which sustained a temporal resolution 
of $\leq 1$ day throughout 1996 June and July. In $\S 2$ we describe the observations and the data reduction procedures, including the intercalibration of ground-based data sets taken at several observatories during the monitoring campaign. In $\S 3$ we present the mean and rms spectra, and line and continuum light curves. A time series analysis based on cross-correlation techniques is described in $\S 4$, yielding estimates of $\sim 5-6$ days for the lags of the $\mathrm{H} \beta$ and $\mathrm{H} \alpha$ emission lines, and shows evidence for wavelengthdependent continuum lags. In $\S 5$ we discuss our results and take the first steps toward interpreting the wavelengthdependent time delays in the context of a simple irradiated accretion disk model of an AGN. The paper concludes with a summary of the results in $\S 6$.

\section{OBSERVATIONS AND DATA REDUCTION}

\subsection{Observations}

From 1996 June 2 to July 30, 54 nights of spectrophotometric observations of NGC 7469 were completed. Approximately 200 spectra spanning $\sim 58$ days were acquired. Most of the spectra were secured on 42 nights with the Wise Observatory's $1 \mathrm{~m}$ telescope and Faint Object Spectroscopic Camera (Kaspi et al. 1996). A 600 line $\mathrm{mm}^{-1}$ grism gave a spectral resolution of $\sim 8 \AA$ over the wavelength range 4016-7841 $\mathrm{A}$. The $10^{\prime \prime} \times 16^{\prime \prime} .8$ slit was aligned at a P.A. of 26.7 in order to observe the active nucleus simultaneously with the $V=11$ mag comparison star $\sim 3^{\prime}$ from NGC 7469 (star 2 of Penston, Penston, \& Sandage 1971). This technique can achieve a relative spectrophotometric accuracy of $\sim 1 \%$, as discussed in $\S 2.2$ (see also Maoz et al. 1991).

Four other spectroscopic data sets were obtained by AGN Watch teams. Of these, two were judged suitable for intercalibration with the Wise data set (see $\$ 2.3$ ). The Ohio State University (OSU) group observed NGC 7469 on 8 nights during the period 1996 May 9 to July 9 with the $1.8 \mathrm{~m}$ Perkins telescope and Bollen and Chivens spectrograph. The 4540-5700 $\AA$ wavelength range was covered with a spectral resolution of $\sim 9 \AA$. A $5^{\prime \prime} \times 7^{\prime \prime} .4$ slit was used throughout the observations. At the Crimean Astrophysical Observatory (CAO), 19 observations were made during 1996 June 11 to October 16 at the Nasmyth focus of the $2.6 \mathrm{~m}$ Shajn telescope. The wavelength range was 4300 $5594 \AA$, the spectral resolution $\sim 8 \AA$. The $3^{\prime \prime} \times 11^{\prime \prime}$ slit was aligned at a P.A. of $90^{\circ}$.

\subsection{Data Reduction}

The OSU and CAO data sets were reduced in a standard fashion and internally flux calibrated using the [O III] emission lines as in previous AGN Watch optical projects. The method of intercalibrating the spectroscopic data sets was first used by Peterson et al. (1991). The physical justification for it is presented in detail in Peterson et al. (1995) and will be discussed in $\S 2.3$. We discuss below reduction of the Wise data set in more detail because this employed nonstandard reduction procedures similar but not identical to those employed in previous Wise Observatory campaigns (Maoz et al. 1990). The Wise data form the backbone of this campaign, and therefore we took additional care to ensure and verify its relative spectrophotometric accuracy approaching $1 \%$.

The CCD spectra were reduced with FIGARO, a general purpose data reduction package maintained by the STARLINK network. Typically, an average of 20 bias frames were used to subtract the constant offset applied to the CCD chip. Short $8 \mathrm{~s}$ exposures of the twilight sky and an incandescent halogen lamp were used to flat-field the data frames. The twilight flats were used to calibrate the spatial illumination pattern along the slit, while the incandescent lamp flats were used to calibrate the pixel-to-pixel sensitivity variations of the CCD detector. Noise models fitted to calibrated data frames gave values for the rms readout noise (0.8 analog-to-digital units [ADU]) and gain (8.4 photons $\mathrm{ADU}^{-1}$ ) of the CCD detector, and were then used to establish error bars on each CCD pixel, and subsequently on the extracted spectra.

The narrow-slit instrumental resolution is $\sim 8 \AA$, but for the wide-slit observations the spectral resolution is increased by the seeing $\left(1^{\prime \prime}\right.$ projects to $\sim 1.8 \AA$ in the dispersion direction). We quantified the seeing by measuring the FWHM of the comparison star profile on each CCD frame, finding FWHMs of $\sim 2.8-3.3$ pixels (5".9-6"9). Subtracting in quadrature the narrow-slit resolution $(2.1$ pixels $=4.4)$, we estimate a seeing range of 3". $9-5$ ". 3 (FWHM).

The galaxy and comparison star spectra were "traced" on the CCD frames with polynomial fits to compensate for optical distortions and differential refraction effects. Spectra were then extracted by summing the counts in $16^{\prime \prime} .8$ extraction windows, after subtracting a sky background fit. Arclamp (HeAr) exposures taken approximately every 20 minutes supplied an accurate wavelength scale, calibrating the small wavelength shifts caused by flexure in the spectrograph. Air-mass corrections were applied to account for continuous extinction using an empirically derived estimate for the extinction as a function of wavelength for the Wise Observatory observing site. Absolute flux calibration was based on observations of BD $+28^{\circ} 4211$ observed on photometric nights.

Atmospheric absorption bands were removed from the AGN spectra with a first-order "B-star" calibration technique (Wade \& Horne 1988). Each comparison star spectrum was divided by a spline fit to normalize its continuum, and all regions outside the telluric absorption bands were set equal to unity. Dividing the AGN spectra by this telluric band spectrum removed the atmospheric absorption bands.

Relative flux calibration was then achieved by calibrating a time- and wavelength-dependent slit loss. An average comparison spectrum was composed from five observations on July 8 , a photometric night. All comparison star spectra were divided by this photometric spectrum, and then thirdorder polynomials were fitted to the ratio spectra, masking any residual absorption features. The polynomial fits represent the wavelength-dependent light losses affecting each spectrum, assuming that the average spectrum is "photometric" and that the nucleus and comparison star experience the same slit losses. The final AGN spectra were produced by dividing by the corresponding polynomial fits. After performing these slit-loss corrections, the comparison star spectra yielded a constant flux to within $\sim 1 \%$. This rms scatter is primarily attributable to the uncertainty in fitting the low-order polynomial and represents a lower limit to the uncertainty in the relative flux calibration. Final light curves were computed by summing the fluxes in a given wavelength region. For emission-line light curves, the subtracted continuum was defined as a straight line fitted to continuum bands on either side of the line. Then when multiple spectra were obtained on a given night, a weighted average with $1 / \sigma^{2}$ weights was computed, where $\sigma$ is the flux 


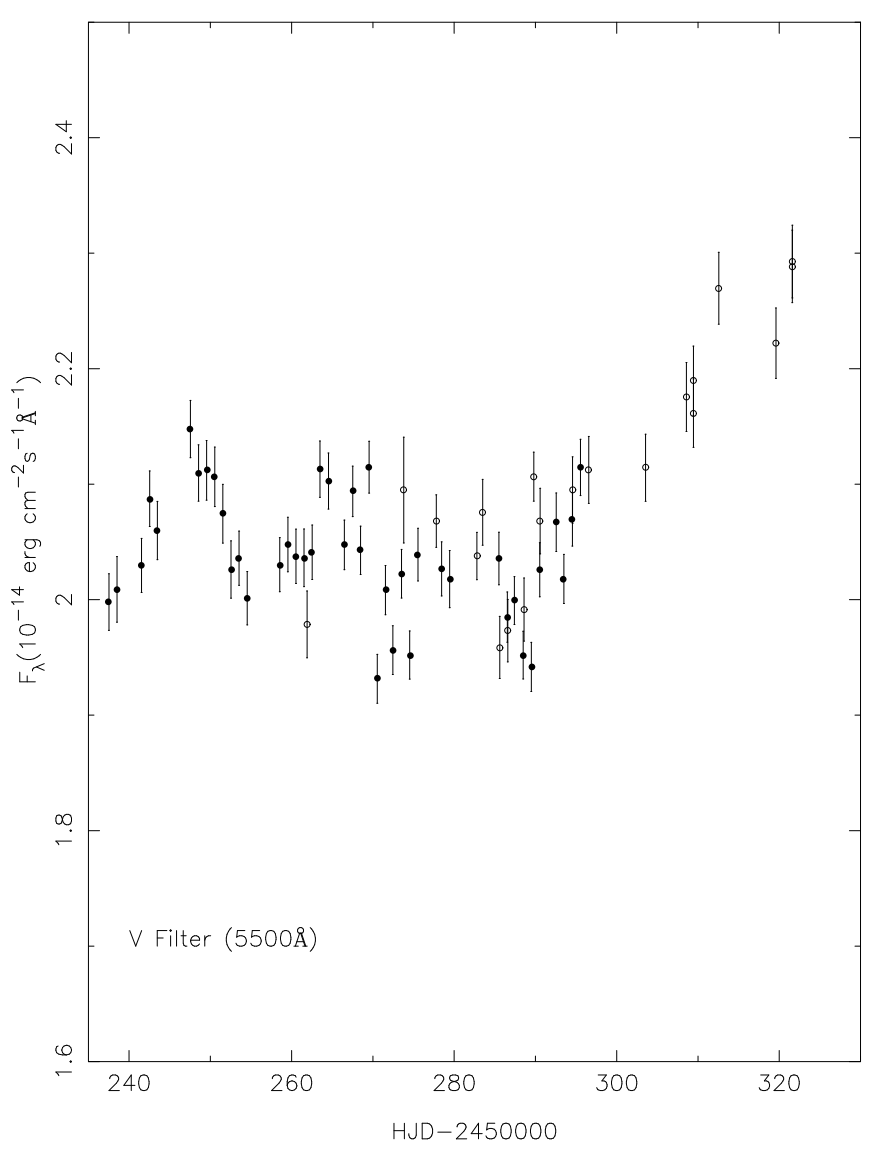

FIG. 1.-Broadband $V$ filter light curve. The open symbols denote photometric observations, while the filled symbols denote synthetic photometry derived from the Wise spectrophotometric data set.

error associated with each pixel. A $1 \%$ calibration error was added in quadrature to that of the error in the weighted mean, to give the final error bars on each measurement.

\subsection{Intercalibration}

A more extensive temporal coverage was achieved by intercalibrating the additional data sets with the reference data set taken at the Wise Observatory. The low amplitude of the variability seen in the Wise data set placed heightened emphasis on high-quality homogeneous data sets overlapping with the intensive monitoring at Wise Observatory. We required the calibrated fluxes to agree to $\leq 3 \%$ with the Wise fluxes. Two data sets met these criteria, the Ohio State University data set (eight spectra), and the CAO data set (19 spectra).

The intercalibration procedure assumes that the flux emitted in the narrow [O $\mathrm{III}$ ] emission lines is constant on the timescales of interest because these low-density lines have a longer recombination time and form at much greater distances from the central source than the broad emission lines. The $[\mathrm{O} \mathrm{III}]$ emission-line flux was computed for each spectrum of the reference data set by summing the flux in the 5025-5103 $\AA$ region, after subtracting a straight line continuum connecting 5000-5020 $\AA$ to 5110-5130 $\AA$. The 194 Wise spectra gave a mean [O III] flux of $(7.5 \pm 0.2) \times 10^{-13}$ ergs $\mathrm{s}^{-1} \mathrm{~cm}^{-2}$. The rms scatter of $\sim 2.7 \%$ indicates the level of stability of the narrow [O III] emission lines.

To validate the accuracy of the relative flux calibration, we used the van Groningen \& Wanders (1992) scaling algo- rithm to scale the [O III] flux of the spectroscopic Wise data set to a constant value (i.e., without the spectrophotometric calibrations). This method uses a template spectrum, which we obtained from a photometric, high signal-to-noise spectrum. The algorithm subtracts the (scaled, shifted, blurred) template spectrum, then fits a low-order function to a given wavelength region, minimizing the $\chi^{2}$ of the residuals. Nightly mean [O III] fluxes were calculated, and their rms scatter of $\sim 2.7 \%$ was consistent with that found from the relative flux calibration based on the comparison star.

The 27 additional spectra from the OSU and CAO data sets were scaled to a constant [O III] flux, using the van Groningen \& Wanders (1992) algorithm, with the mean of the 194 Wise spectra as the template spectrum. Subsequent stages of intercalibration involved correcting for differences in the light loss suffered by various AGN components due to aperture size differences among the data sets. A wavelength-independent multiplicative correction factor accounts for apertures admitting different fractions of the point-spread function and of the extended narrow-line emission region. The point-spread function describes the surface brightness distribution of both the broad emission lines and the nuclear continuum source. A wavelengthdependent additive correction corrects for apertures admitting different fractions of the starlight from the host galaxy. This additive correction factor applies to continuum regions and is a function of wavelength due to the wavelength dependence of the host galaxy starlight. The exact procedure is described in some detail elsewhere (Peterson et al. 1995) and will not be repeated here.

The light curves extracted from the 4840-4890 A, 4900$5000 \AA$, and $5160-5220 \AA$ wavelength regions of the OSU and CAO data sets were intercalibrated successfully. At longer wavelengths intercalibration proved problematic due to the errors of the intercalibrated fluxes becoming comparable to the amplitude of variability observed. Formal errors were assigned to the intercalibrated data sets based on the accuracy to which we could compute the correction factors previously described. In both cases the error bars assigned were $\leq 2 \%$.

\subsection{Photometric Observations}

Two sets of differential photometric observations were obtained. The Behlen Observatory group made five Johnson $V$ filter observations through a circular aperture of diameter 13"7 during 1996 June 27-July 25. The comparison star used was HST Guide Star Catalog 1160585 $(V=13.45 \mathrm{mag})$. The Osservatorio Astronomico di Bologna group made 15 Johnson $V$ filter observations through a circular aperture of diameter 18" during 1996 July 19-August 26 . The companion star used was star 4 $(V=12.67 \mathrm{mag})$ of Penston et al. (1971).

The Behlen data set was internally intercalibrated with that of the Bologna Observatory data, as these observations showed most overlap with the intensive monitoring period. Differential magnitudes were transformed to apparent magnitudes using the apparent magnitudes of the comparison stars, as derived from the literature. Observations made within \pm 1 day were compared, and then $0.45 \pm 0.03 \mathrm{mag}$ was added to the Behlen observations to internally intercalibrate the two data sets. The resulting apparent magnitudes were then transformed to fluxes by (Lang 1980)

$$
\log F_{\lambda}(5500 \AA)=-0.4 V-8.42 \text {. }
$$


These fluxes are shown in Figure 1, along with synthetic photometric fluxes derived from the Wise spectrophotometry. Similar structure is seen in both photometric and synthetic photometric fluxes, confirming the robustness of the spectrophotometric calibrations. The difference in flux levels most likely results from different aperture sizes admitting different fractions of the host galaxy.

\section{RESULTS}

\subsection{Mean and Root Mean Square Spectra}

The mean and root mean square (rms) of 194 spectra (the complete Wise data set) are shown in Figure 2. The rms spectrum characterizes the variable component of the spectrum.

Salient features in the mean spectrum are broad $\mathrm{H} \gamma, \mathrm{H} \beta$, and $\mathrm{H} \alpha$ emission lines, observed at wavelengths of $\sim 4407$, 4940 , and $6667 \AA$. Broad He I $\lambda 5876$ and He II $\lambda 4686$ are observed as low-contrast features. Forbidden narrow lines of [O III], [O I], and [S II] are observed at $\sim 5036,5086$, 6400,6824 , and $6836 \AA$, respectively. Typical FWHMs are $\sim 2000 \mathrm{~km} \mathrm{~s}^{-1}$ for the broad lines and $500 \mathrm{~km} \mathrm{~s}^{-1}$ for the narrow lines. Broadband features from blended Fe II multiplets are observed at $\sim 4650$ and $5350 \AA$. The mean spec-

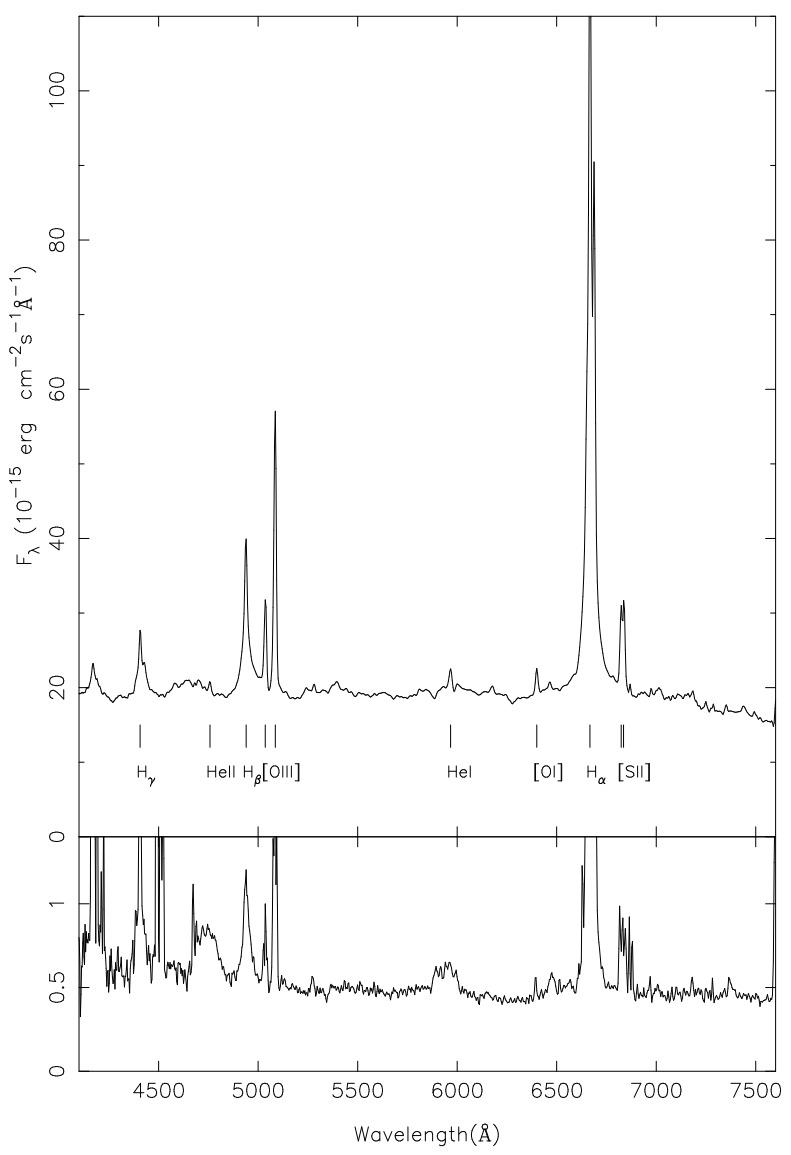

FIG. 2.-Top panel: Mean spectrum of NGC 7469. Bottom panel: The rms spectrum of NGC 7469, characterizing or showing the spectrum of the variable component. Imperfect wavelength calibration results in spurious peaks near each of the narrow lines. trum declines in flux toward longer wavelengths. This may be a result of the relatively blue component of the starburst ring that surrounds NGC 7469 at a radius of $\sim 8-10^{\prime \prime}$ (Salamanca et al. 1995). The more rapid decline of continuum flux longward of $7200 \AA$ results from imperfect calibration of the sensitivity of the CCD detector.

The rms spectrum (lower panel of Fig. 2) confirms that low-amplitude line and continuum variability was detected during the intensive monitoring period. The continuum varies by $\sim 4 \%$ at $4800 \AA$ and only $\sim 2 \%$ at $7000 \AA$. The broad He II $\lambda 4686$ emission is not clearly visible in the mean spectrum due to blending with $\mathrm{Fe}$ II emission, but the rms spectrum reveals a broad peak with rms variations up to $\sim 5 \%$ of the corresponding mean flux. Imperfect wavelength calibration results in spurious peaks in the rms spectrum near each of the narrow lines, but this does not seriously affect the continuum and broad-line features.

The host galaxy contribution was estimated in the following way: Two spectra were extracted from a high signal-tonoise data frame, with extraction windows of $16^{\prime \prime} .8$ and $42^{\prime \prime}$. Subtracting the smaller from the larger aperture spectrum led to an estimate of the host galaxy spectrum in two $10^{\prime \prime}$ $\times 12^{\prime \prime} .6$ apertures straddling the $10^{\prime \prime} \times 16^{\prime \prime} .8$ window. We assume that the spectral shape of the host galaxy is the same in both apertures, but apply a scale factor (0.79) to account for the smaller solid angle and higher surface brightness of the host galaxy in the $10^{\prime \prime} \times 16^{\prime \prime} 8$ aperture. From this we estimate that the host galaxy contaminates the mean spectrum by $\sim 18 \%$ or $3.7 \pm 0.7 \mathrm{mJy}$ at $5400 \AA$. This compares favorably with an independent estimate of $3.2 \pm 0.6 \mathrm{mJy}$, from the results presented by Malkan \& Filippenko (1983).

\subsection{Light Curves}

Figure 3 presents the $4840-4890 \AA$ continuum and $\mathrm{H} \beta$ light curves for the complete monitoring campaign, May 9 to Oct 17. Observed wavelengths and fluxes are used throughout this paper, with no correction for the redshift $(z=0.0164)$ of the object. Emission-line light curves were constructed by summing the flux in a given wavelength region after subtracting a pseudocontinuum defined as a straight line fitted to the continuum about the wavelength region of interest. The light curves are tabulated in Table 1. Referring to Figure 3, both light curves begin with an $\sim 18 \%$ decrease in flux over 12 days to a lower flux level, where $\sim 10 \%$ flux fluctuations occur on timescales of $\sim 1$ week during the 60 day intensive monitoring period. There follows a steep rise in flux of $\sim 40 \%$ over the next 2 months, leveling out for the last $\sim 30$ days of the monitoring campaign.

Light curves for the intensive monitoring period are presented in Figure 4. Descending from the top panel, the wavelength regions are 4840-4890 $\AA, 6925-7000 \AA, \mathrm{H} \beta$ (4900-5000 $\AA)$, and $\mathrm{H} \alpha(6630-6730 \AA)$. The $1315 \AA$ light curve from the IUE observations (Wanders et al. 1997) is scaled and vertically shifted to fit each of the optical light curves and is shown by the overplotted solid line in Figure 4.

All the continuum regions show the same general up and down undulations in flux, with a quasi period of $\sim 17$ days. The full range of the variations, as defined by $\left(F_{\max } /\right.$ $\left.F_{\text {min }}-1\right)$, is $\sim 0.14$ at $4865 \AA$ and $\sim 0.11$ at $6925 \AA$. A trend of decreasing flux throughout the intensive monitoring 


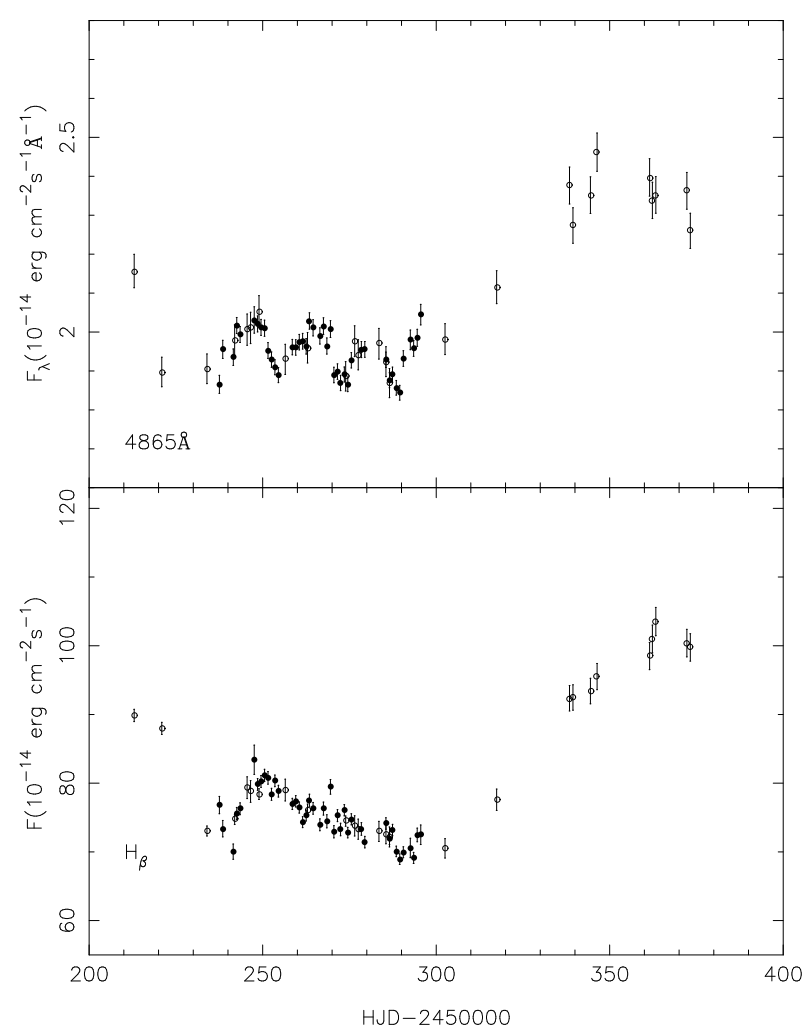

FIG. 3.-Light curves for the entire monitoring campaign, 1996 May 9-October 16. Top panel: $4865 \AA ̊$ light curve. Bottom panel: $\mathrm{H} \beta$ light curve. The open symbols denote intercalibrated data. Discussion as in the text.

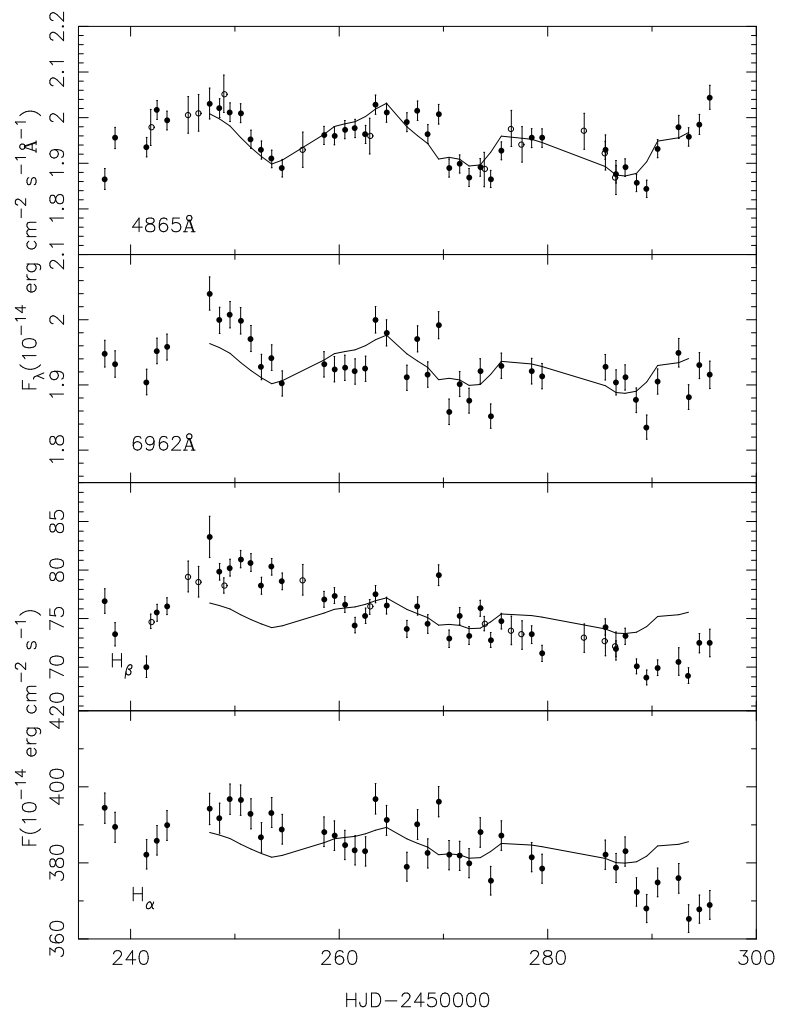

FIG. 4.- Light curves for the intensive monitoring period, June 2-July 31. Wavelength regions as labeled in the figure. The open symbols denote intercalibrated data. Line and continuum fluxes in units of $10^{-14} \mathrm{ergs}$ $\mathrm{cm}^{-2} \mathrm{~s}^{-1}$ and $10^{-14}$ ergs $\mathrm{cm}^{-2} \mathrm{~s}^{-1} \AA^{-1}$. period in both emission-line fluxes is evident. The full range of variability, primarily attributable to this trend, is $\sim 0.20$ for the $\mathrm{H} \beta$ light curve and $\sim 0.08$ for the $\mathrm{H} \alpha$ light curve. A less pronounced decline in the continuum fluxes is also evident.

\subsection{Variability Characteristics}

We use four parameters to characterize the line and continuum variability of the light curves presented in Figure 4. The mean $(\bar{F})$ and rms flux $\left(\sigma_{F}\right)$ are defined in the standard fashion. The ratio of the maximum to the minimum flux, $R_{\max }$, and the amplitude of the intrinsic variability relative to the mean flux, $F_{\text {var }}$, corrected for the measurement errors $\varepsilon$, are defined by

$$
F_{\mathrm{var}}=\frac{1}{\bar{F}} \sqrt{\left(\sigma_{F}^{2}-\Delta^{2}\right)},
$$

where $\Delta^{2}$ is the mean square of the measurement uncertainties, defined as

$$
\Delta^{2}=\frac{1}{N} \sum_{i=1}^{N} \varepsilon_{i}^{2}
$$

where $N$ is the number of data points in the light curve (Rodríguez-Pascual et al. 1997).

The results are presented in Table 2, in which column (1) is the light curve considered, column (2) is the associated number of data points $N$, column (3) is the mean flux $\bar{F}$, column (4) is the rms flux $\sigma_{F}$, column (5) is $F_{\text {var }}$, and column (6) is $R_{\max }$. The amplitude of the intrinsic variations $F_{\text {var }}$ is $\sim 3 \%$ at $4865 \AA$ and $\sim 2 \%$ at $6962 \AA$.

\section{CROSS-CORRELATION ANALYSIS}

A cross-correlation analysis was carried out to quantify time delays for the emission lines and to investigate wavelength-dependent time delays in the continuum. Two algorithms were employed to compute cross-correlation functions (CCFs): the interpolation CCF (ICCF) of Gaskell \& Sparke (1986) (Gaskell \& Peterson 1987), as implemented by White $\&$ Peterson (1994), and the $Z$-transformed discrete correlation function algorithm (ZDCF) of Alexander (1998). For our well-sampled data the results recovered by the two algorithms are very similar.

Figure 5 presents the CCFs. Earlier reports of small ( $\sim 0.3$ day) wavelength-dependent time delays in the UV data from this same monitoring campaign (Wanders et al. 1997) led us to investigate time delays between the UV and optical data. All the optical light curves are cross correlated with the $1315 \AA$ light curve, and only data points within the intensive monitoring period are included in the analysis. Each panel contains the CCFs for both methods. The ICCF results are presented as solid lines, the ZDCF results as the points with error bars. The CCFs appear shifted by 1-2 days for the continuum and 4-6 days for the lines.

The autocorrelation function (ACF) for the $1315 \AA$ light curve is overplotted in all panels as the dashed line. The FWHM of the ACF is $\sim 4.9$ days, indicative of the variability timescale of the $1315 \AA$ variations. The CCFs, presented in Figure 5, are broader than the $1315 \AA$ ACF. This may suggest that the optical responses extend over a range of time delays.

Table 3 summarizes our cross-correlation results. Column (1) is the wavelength band considered. Columns (2) and (3) detail the centroid of the CCF, $\tau_{\text {cen }}$, as determined 
TABLE 1

Light CuRves

\begin{tabular}{|c|c|c|c|c|}
\hline HJD - 2,450,000 & $4865 \AA$ & $6962 \AA$ & $\mathrm{H} \beta$ & $\mathrm{H} \alpha$ \\
\hline $212.973^{\mathrm{a}}$ & $2.16 \pm 0.04$ & & $89.86 \pm 0.90$ & \\
\hline $220.944^{\mathrm{a}}$. & $1.90 \pm 0.04$ & $\ldots$ & $87.97 \pm 0.88$ & $\ldots$ \\
\hline $233.952^{\mathrm{a}}$ & $1.91 \pm 0.04$ & & $73.04 \pm 0.73$ & \\
\hline $237.53 \ldots$ & $1.87 \pm 0.02$ & $1.95 \pm 0.02$ & $76.81 \pm 1.26$ & $394.39 \pm 4.00$ \\
\hline 238.51 . & $1.96 \pm 0.02$ & $1.93 \pm 0.02$ & $73.38 \pm 1.20$ & $389.36 \pm 3.95$ \\
\hline 241.54 & $1.94 \pm 0.02$ & $1.90 \pm 0.02$ & $70.04 \pm 1.11$ & $382.25 \pm 3.85$ \\
\hline $241.93^{\mathrm{a}}$ & $1.98 \pm 0.04$ & & $74.72 \pm 0.75$ & \\
\hline 242.54 . & $2.02 \pm 0.02$ & $1.95 \pm 0.02$ & $75.60 \pm 0.87$ & $385.94 \pm 3.87$ \\
\hline 243.49 . & $1.99 \pm 0.02$ & $1.96 \pm 0.02$ & $76.27 \pm 0.87$ & $389.84 \pm 3.92$ \\
\hline $245.53^{\mathrm{b}}$ & $2.01 \pm 0.04$ & $\ldots$ & $79.34 \pm 1.59$ & $\ldots$ \\
\hline $246.52^{\mathrm{b}}$ & $2.01 \pm 0.04$ & 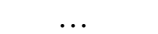 & $78.80 \pm 1.58$ & \\
\hline 247.58 . & $2.03 \pm 0.03$ & $2.04 \pm 0.03$ & $83.41 \pm 2.13$ & $394.20 \pm 4.13$ \\
\hline 248.54 . & $2.02 \pm 0.02$ & $2.00 \pm 0.02$ & $79.82 \pm 0.86$ & $391.78 \pm 3.93$ \\
\hline $248.95^{\mathrm{a}}$ & $2.05 \pm 0.04$ & & $78.40 \pm 0.78$ & \\
\hline 249.54 . & $2.01 \pm 0.02$ & $2.01 \pm 0.02$ & $80.25 \pm 0.87$ & $396.76 \pm 3.98$ \\
\hline 250.55 . & $2.01 \pm 0.02$ & $2.00 \pm 0.02$ & $81.13 \pm 0.89$ & $396.51 \pm 3.98$ \\
\hline 251.54 & $1.95 \pm 0.02$ & $1.97 \pm 0.02$ & $80.78 \pm 0.91$ & $392.90 \pm 3.95$ \\
\hline 252.55 & $1.93 \pm 0.02$ & $1.93 \pm 0.02$ & $78.38 \pm 0.87$ & $386.70 \pm 3.89$ \\
\hline 253.53 . & $1.91 \pm 0.02$ & $1.94 \pm 0.02$ & $80.33 \pm 0.85$ & $393.28 \pm 3.94$ \\
\hline 254.54 . & $1.89 \pm 0.02$ & $1.90 \pm 0.02$ & $78.83 \pm 0.86$ & $388.86 \pm 3.90$ \\
\hline $256.51^{\mathrm{b}}$ & $1.93 \pm 0.04$ & & $79.90 \pm 1.58$ & \\
\hline 258.54 . & $1.96 \pm 0.02$ & $1.93 \pm 0.02$ & $76.99 \pm 0.82$ & $388.20 \pm 3.89$ \\
\hline 259.54 & $1.96 \pm 0.02$ & $1.92 \pm 0.02$ & $77.36 \pm 0.82$ & $387.15 \pm 3.88$ \\
\hline 260.54 . & $1.97 \pm 0.02$ & $1.93 \pm 0.02$ & $76.45 \pm 0.81$ & $384.72 \pm 3.86$ \\
\hline 261.53 . & $1.98 \pm 0.02$ & $1.92 \pm 0.02$ & $74.33 \pm 0.80$ & $383.30 \pm 3.85$ \\
\hline 262.53 . & $1.96 \pm 0.02$ & $1.92 \pm 0.02$ & $75.29 \pm 0.80$ & $383.03 \pm 3.84$ \\
\hline $262.93^{\mathrm{a}}$ & $1.96 \pm 0.04$ & & $76.20 \pm 0.76$ & \\
\hline 263.48 & $2.03 \pm 0.02$ & $2.00 \pm 0.02$ & $77.52 \pm 0.88$ & $396.84 \pm 3.98$ \\
\hline 264.54 . & $2.01 \pm 0.02$ & $1.98 \pm 0.02$ & $76.34 \pm 0.86$ & $391.19 \pm 3.93$ \\
\hline 266.50 . & $1.99 \pm 0.02$ & $1.91 \pm 0.02$ & $73.94 \pm 0.87$ & $379.02 \pm 3.80$ \\
\hline 267.52 . & $2.02 \pm 0.02$ & $1.97 \pm 0.02$ & $76.31 \pm 0.94$ & $390.09 \pm 3.92$ \\
\hline 268.50 . & $1.96 \pm 0.02$ & $1.92 \pm 0.02$ & $74.44 \pm 0.96$ & $382.49 \pm 3.84$ \\
\hline $269.54 \ldots$ & $2.01 \pm 0.02$ & $1.99 \pm 0.02$ & $79.47 \pm 1.07$ & $396.11 \pm 3.99$ \\
\hline 270.54 . & $1.89 \pm 0.02$ & $1.86 \pm 0.02$ & $72.93 \pm 0.89$ & $382.03 \pm 3.86$ \\
\hline $271.55 \ldots$ & $1.90 \pm 0.02$ & $1.90 \pm 0.02$ & $75.27 \pm 0.87$ & $381.85 \pm 3.84$ \\
\hline 272.45 . & $1.87 \pm 0.02$ & $1.88 \pm 0.02$ & $73.26 \pm 0.92$ & $379.91 \pm 3.82$ \\
\hline 273.53 . & $1.89 \pm 0.02$ & $1.92 \pm 0.02$ & $76.06 \pm 0.82$ & $388.00 \pm 3.89$ \\
\hline $273.91^{\mathrm{a}}$ & $1.89 \pm 0.04$ & & $74.48 \pm 0.74$ & \\
\hline $274.53 \ldots$ & $1.87 \pm 0.02$ & $1.85 \pm 0.02$ & $72.80 \pm 0.77$ & $375.32 \pm 3.76$ \\
\hline 275.55 . & $1.93 \pm 0.02$ & $1.93 \pm 0.02$ & $74.74 \pm 0.80$ & $387.17 \pm 3.88$ \\
\hline $276.51^{\mathrm{b}}$ & $1.98 \pm 0.04$ & $\ldots$ & $73.78 \pm 1.48$ & $\ldots$ \\
\hline $277.53^{\mathrm{b}}$ & $1.94 \pm 0.04$ & & $73.30 \pm 1.47$ & \\
\hline 278.46 . & $1.95 \pm 0.02$ & $1.92 \pm 0.02$ & $73.34 \pm 0.91$ & $381.52 \pm 3.85$ \\
\hline $279.45 \ldots$ & $1.96 \pm 0.02$ & $1.91 \pm 0.02$ & $71.42 \pm 0.84$ & $378.49 \pm 3.81$ \\
\hline $283.50^{\mathrm{b}}$ & $1.97 \pm 0.04$ & & $72.98 \pm 1.46$ & \\
\hline 285.51 & $1.93 \pm 0.02$ & $1.93 \pm 0.02$ & $74.14 \pm 0.83$ & $382.17 \pm 3.84$ \\
\hline $285.53^{\mathrm{b}}$ & $1.92 \pm 0.04$ & $\ldots$ & $72.62 \pm 1.45$ & $\ldots$ \\
\hline $286.54^{\mathrm{b}}$ & $1.87 \pm 0.04$ & & $72.17 \pm 1.44$ & \\
\hline 286.55 . & $1.88 \pm 0.02$ & $1.90 \pm 0.02$ & $71.88 \pm 0.82$ & $378.68 \pm 3.81$ \\
\hline 287.42 . & $1.89 \pm 0.02$ & $1.91 \pm 0.02$ & $73.17 \pm 0.83$ & $382.98 \pm 3.84$ \\
\hline $288.51 \ldots$ & $1.86 \pm 0.02$ & $1.88 \pm 0.02$ & $70.07 \pm 0.77$ & $372.36 \pm 3.74$ \\
\hline 289.49 . & $1.84 \pm 0.02$ & $1.84 \pm 0.02$ & $68.94 \pm 0.77$ & $367.97 \pm 3.70$ \\
\hline $290.54 \ldots \ldots \ldots \ldots$ & $1.93 \pm 0.02$ & $1.91 \pm 0.02$ & $69.94 \pm 0.80$ & $374.86 \pm 3.77$ \\
\hline $292.56 \ldots$ & $1.98 \pm 0.03$ & $1.95 \pm 0.02$ & $70.58 \pm 1.42$ & $375.93 \pm 3.91$ \\
\hline $293.51 \ldots \ldots \ldots \ldots$ & $1.96 \pm 0.02$ & $1.88 \pm 0.02$ & $69.12 \pm 0.81$ & $365.35 \pm 3.66$ \\
\hline $294.54 \ldots$ & $1.99 \pm 0.02$ & $1.93 \pm 0.02$ & $72.46 \pm 1.00$ & $367.85 \pm 3.71$ \\
\hline $295.55 \ldots$ & $2.04 \pm 0.03$ & $1.92 \pm 0.02$ & $72.49 \pm 1.42$ & $368.96 \pm 3.78$ \\
\hline $302.55^{\mathrm{b}}$ & $1.98 \pm 0.04$ & $\ldots$ & $70.51 \pm 1.41$ & $\ldots$ \\
\hline $317.43^{\mathrm{b}}$. & $2.11 \pm 0.04$ & $\ldots$ & $77.59 \pm 1.55$ & $\ldots$ \\
\hline $338.43^{\mathrm{b}}$ & $2.38 \pm 0.05$ & $\ldots$ & $92.36 \pm 1.85$ & $\ldots$ \\
\hline $339.42^{\mathrm{b}}$ & $2.27 \pm 0.05$ & $\ldots$ & $92.47 \pm 1.85$ & $\ldots$ \\
\hline $344.45^{\mathrm{b}}$. & $2.35 \pm 0.05$ & $\ldots$ & $93.41 \pm 1.87$ & .. \\
\hline $346.36^{\mathrm{b}}$. & $2.46 \pm 0.05$ & $\ldots$ & $95.51 \pm 1.91$ & $\ldots$ \\
\hline $361.49^{\mathrm{b}}$. & $2.40 \pm 0.05$ & $\ldots$ & $98.49 \pm 1.97$ & $\ldots$ \\
\hline $362.30^{\mathrm{b}}$ & $2.34 \pm 0.05$ & $\ldots$ & $101.0 \pm 2.0$ & $\ldots$ \\
\hline $363.29^{\mathrm{b}}$. & $2.35 \pm 0.05$ & $\ldots$ & $103.5 \pm 2.1$ & $\ldots$ \\
\hline $372.22^{\mathrm{b}} \ldots \ldots \ldots$ & $2.36 \pm 0.05$ & $\ldots$ & $100.4 \pm 2.0$ & $\ldots$ \\
\hline $373.22^{b} \ldots \ldots \ldots \ldots$ & $2.26 \pm 0.05$ & $\ldots$ & $99.76 \pm 2.00$ & $\ldots$ \\
\hline
\end{tabular}

NotE.-Continuum flux in units of $10^{-14} \mathrm{ergs} \mathrm{cm}^{-2} \mathrm{~s}^{-1} \AA^{-1}$. Emission-line fluxes in units of $10^{-14} \mathrm{ergs} \mathrm{cm}^{-2} \mathrm{~s}^{-1}$.

${ }^{a}$ OSU data.

${ }^{\mathrm{b}} \mathrm{CAO}$ data. 
TABLE 2

\begin{tabular}{cccccc}
\multicolumn{7}{c}{ VARIABILITY PARAMETERS } \\
\hline \hline $\begin{array}{c}\text { Light Curve } \\
(1)\end{array}$ & $\begin{array}{c}N \\
(2)\end{array}$ & $\begin{array}{c}\bar{F}^{\mathrm{a}} \\
(3)\end{array}$ & $\begin{array}{c}\sigma_{F}^{\mathrm{a}} \\
(4)\end{array}$ & $\begin{array}{c}F_{\text {var }} \\
(5)\end{array}$ & $\begin{array}{c}R_{\max } \\
(6)\end{array}$ \\
\hline $4865 \AA \ldots \ldots$. & 54 & 1.95 & 0.05 & 0.02 & 1.11 \\
$6962 \AA \ldots \ldots$. & 42 & 1.93 & 0.04 & 0.02 & 1.11 \\
$\mathrm{H} \beta \ldots \ldots \ldots$. & 54 & 7.52 & 0.34 & 0.04 & 1.21 \\
$\mathrm{H} \alpha \ldots \ldots \ldots$. & 42 & 38.4 & 0.84 & 0.02 & 0.09 \\
\hline
\end{tabular}

a Units are $10^{-14} \mathrm{ergs} \mathrm{cm}^{-2} \mathrm{~s}^{-1} \AA^{-1}$ for continuum fluxes and $10^{-13} \mathrm{ergs} \mathrm{cm}^{-2} \mathrm{~s}^{-1}$ for line fluxes.

by the ICCF and ZDCF algorithms. The ICCF centroid is calculated over all points above 0.8 times the maximum cross-correlation amplitude, whereas the ZDCF centroid is computed from all points near the peak with a cross-

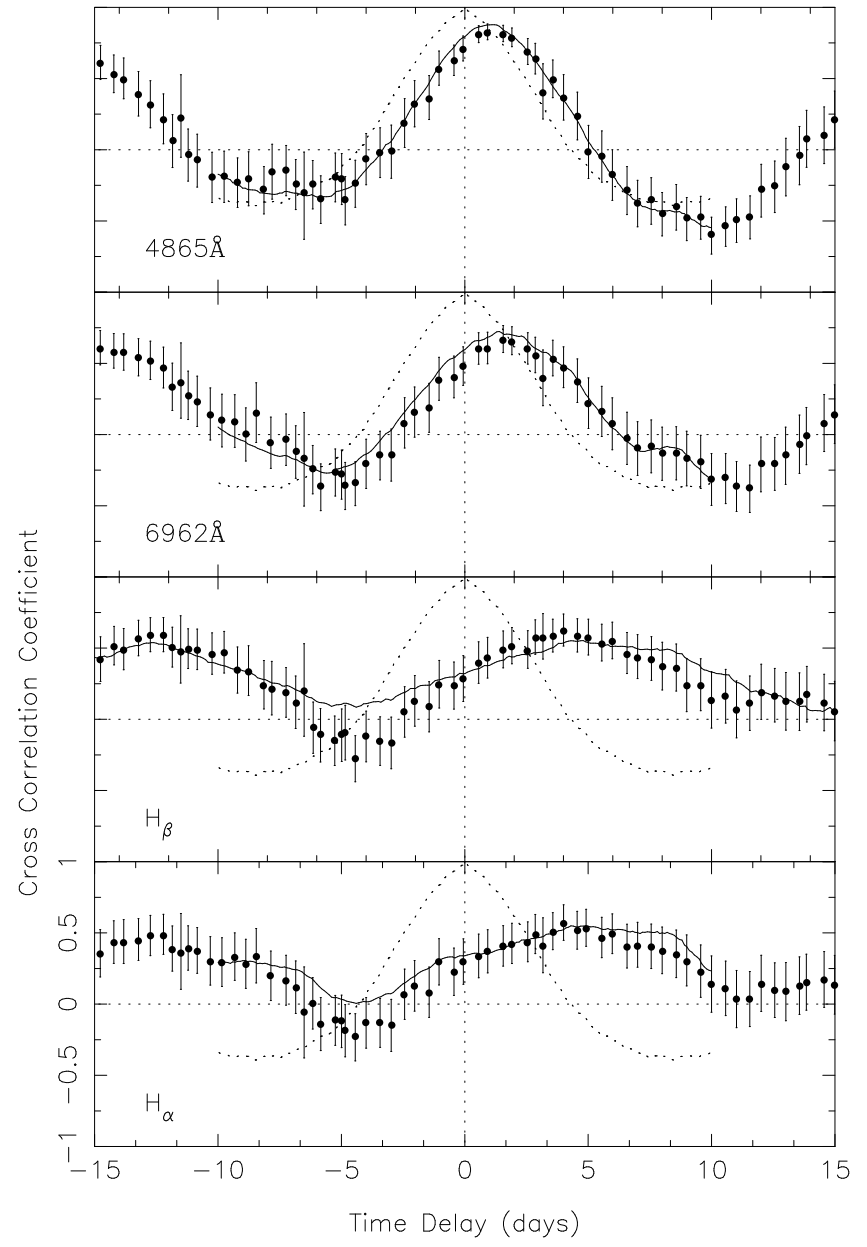

FIG. 5.-Results of cross-correlation analysis. The solid line represents the ICCF CCF; the dashed line is the $1315 \AA$ ACF; and the data points with error bars present the ZDCF CCF. A full discussion is given in the text. correlation coefficient above half that of the peak. Columns (4) and (5), similarly, report the time delay, $\tau_{\text {peak }}$, found from the peak of the CCF. Columns (6) and (7) report the maximum cross-correlation coefficient, $r_{\max }$. Column (8) reports the FWHM of the CCF, as determined from the ICCF algorithm.

The centroid of the ICCF is $1.0 \pm 0.3$ days at $4865 \AA$, and $1.5 \pm 0.7$ days at $6962 \AA$. The corresponding strength of the correlation, $r_{\max }$, defined by the highest cross-correlation coefficient is 0.9 at $4845 \AA$ and 0.7 at $6962 \AA$. The error estimates for the centroid of the ICCF are computed using a "quasi-bootstrap method," as described in $\S 4.3$. The crosscorrelation results from the two methods are always in agreement to within the quoted uncertainties.

\subsection{Emission-Line Time Delays}

The bottom two panels of Figure 5 show the cross correlation of the $1315 \AA$ light curve with the mean $\mathrm{H} \beta$ and $\mathrm{H} \alpha$ light curves (shown in Fig. 4). For $\mathrm{H} \beta$ and $\mathrm{H} \alpha$, the ICCF peaks at $4.3 \pm 0.4$ days and $4.8 \pm 0.5$ days, respectively, and the cross-correlation coefficients are $\sim 0.6$. From Figure 5 and Table 3 we conclude that both emission-line CCFs are broader than the continuum CCFs. This suggests that the Balmer line gas is responding to the continuum variations with a range of time delays.

\subsection{Continuum Time Delays}

The CCFs shown in Figure 5 indicate a UV-to-optical time delay of $\sim 1-2$ days. To investigate further we bin the 194 Wise spectra into $40 \AA$ wide bins, over the wavelength range 4040-7720 $\AA$, and cross correlate each of the resulting 94 mean light curves with the $1315 \AA$ light curve. Subsequently, we computed the centroid lag for each wavelength bin. The results are presented in the upper panel of Figure 6. The ICCF centroid increases with wavelength, from $\sim 1$ day at $4800 \AA$ to $\sim 2$ days at $7500 \AA$. The error bars on the data points were determined using a quasi-bootstrap method to be discussed shortly. The maximum crosscorrelation coefficient $r_{\max }$, shown in the lower panel of Figure 6, is around $0.6-0.8$ at most wavelengths, but with some dips near the wavelengths of the emission lines. The time delay increases above the overall trend near the emission lines because here the light curve has a mix of continuum and line flux. The lines respond with longer time delays, and the net delay is a mix of emission line and continuum time delays. This mixing also produces a decrease in $r_{\max }$. Dips in $r_{\max }$ also occur near narrow [O III] and [S II] lines due to poor wavelength shifts. Figure 6 strengthens the evidence for wavelength-dependent time

TABLE 3

Cross-Correlation Results

\begin{tabular}{|c|c|c|c|c|c|c|c|}
\hline \multirow[b]{2}{*}{$\begin{array}{l}\text { BAND } \\
\text { (1) }\end{array}$} & \multicolumn{2}{|c|}{$\tau_{\text {cen }}$ (days) } & \multicolumn{2}{|c|}{$\tau_{\text {peak }}$ (days) } & \multicolumn{2}{|c|}{$r_{\max }$} & \multirow{2}{*}{$\begin{array}{c}\text { FWHM ICCF } \\
\text { (days) } \\
\text { (8) }\end{array}$} \\
\hline & $\begin{array}{c}\text { ICCF } \\
\text { (2) }\end{array}$ & $\begin{array}{c}\text { ZDCF } \\
\text { (3) }\end{array}$ & $\begin{array}{c}\text { ICCF } \\
\text { (4) }\end{array}$ & $\begin{array}{c}\text { ZDCF } \\
(5)\end{array}$ & $\begin{array}{c}\text { ICCF } \\
\text { (6) }\end{array}$ & $\begin{array}{c}\text { ZDCF } \\
\text { (7) }\end{array}$ & \\
\hline $4865 \AA$. & $1.0 \pm 0.3$ & 1.2 & $1.1 \pm 0.1$ & $0.9_{-0.5}^{+0.7}$ & 0.9 & 0.8 & 5.1 \\
\hline $6962 \AA \ldots . . .$. & $1.5 \pm 0.7$ & 1.9 & $1.4 \pm 0.2$ & $1.6_{-0.8}^{+0.8}$ & 0.7 & 0.7 & 6.0 \\
\hline $\mathrm{H} \beta \ldots \ldots \ldots$ & $5.4 \pm 0.8$ & 4.3 & $4.8 \pm 0.5$ & $4.0_{-1.0}^{+1.4}$ & 0.6 & 0.6 & 11.9 \\
\hline $\mathrm{H} \alpha \ldots \ldots \ldots$ & $5.6 \pm 1.3$ & 4.4 & $4.3 \pm 0.4$ & $4.0_{-1.7}^{+2.0}$ & 0.5 & 0.6 & 10.9 \\
\hline
\end{tabular}



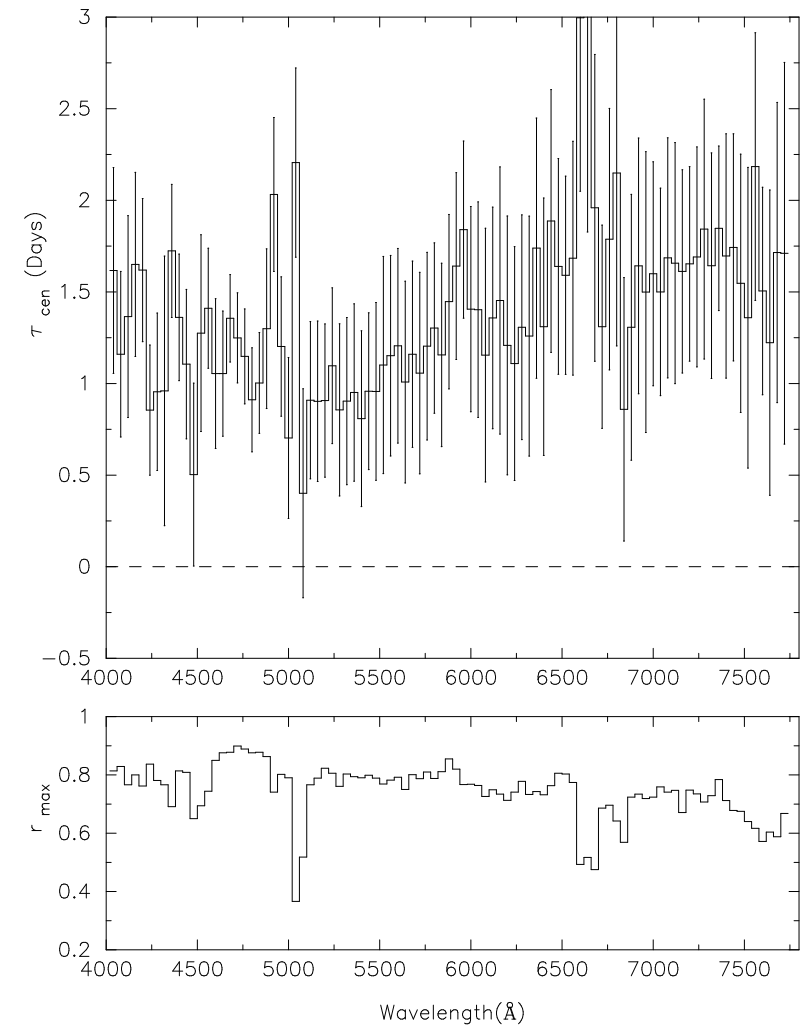

FIG. 6.-Wavelength-dependent time delays, binned to $40 \AA$ A. Top panel: Data points connected by the solid line denote the centroid of the CCF using the ICCF algorithm. The errors were assigned via a quasi-bootstrap method as described in the text. Bottom panel: Data points connected by the solid line detail the maximum cross-correlation coefficient of the ICCF algorithm.

delays between the UV and optical continua and confirms the time delays observed from Figure 5 and Table 3.

\subsection{Significance of the Wavelength-dependent Continuum Lags}

Given the implications of our result, we considered carefully whether the observed lags could arise from an instrumental effect. The most plausible effect we identified is a modulation of the contaminating red galaxy light induced by seeing variations or pointing errors. Since the host galaxy is redder than the AGN, suitable seeing and pointing errors could in principle produce variations that mimic by accident a time delay increasing toward the red end of the spectrum. However, the following considerations convince us that this is not the case.

Figure 4 shows that the rms difference between the continuum light curves and the scaled and shifted $1315 \AA$ light curve is $\sim 5 \%$. Therefore $\sim 5 \%$ perturbations of the optical

TABLE 4

ERROR ESTIMATES FOR CCF LAGS

\begin{tabular}{cccc}
\hline \hline $\begin{array}{c}\lambda_{\text {center }} \\
(\AA)\end{array}$ & $\begin{array}{c}\text { Number of Bins } \\
(1)\end{array}$ & $\begin{array}{c}\langle\text { Lag } \\
\text { (days) } \\
(3)\end{array}$ & $\begin{array}{c}\sigma \\
(\text { days }) \\
(4)\end{array}$ \\
\hline $5260 \ldots \ldots$ & 4 & $0.94 \pm 0.06$ & 0.11 \\
$7060 \ldots \ldots$ & 4 & $1.54 \pm 0.06$ & 0.12 \\
$7420 \ldots \ldots$ & 4 & $1.71 \pm 0.06$ & 0.12 \\
\hline
\end{tabular}

continuum are needed to produce the time delay. Given our estimate in $\S 3.1$ of $\sim 18 \%$ for the host galaxy contribution, a $\sim 27 \%$ change in the host galaxy contamination is required to change the optical continuum by $\sim 5 \%$. To assess whether so large a change in the level of contamination could be introduced by fluctuations in the seeing, we modeled how the light entering our $10^{\prime \prime} \times 16^{\prime \prime} 8$ aperture from the nucleus and host galaxy may depend on seeing. The seeing varied from $\sim 3^{\prime \prime} .9-5^{\prime \prime} .3$, but in this range the maximum aperture loss in light from the two components was $\sim 1 \%$. Indeed on July 3 when the seeing was most variable, there were no significant $(\geq 1 \%)$ fluctuations. Similarly, misguiding and miscentering (pointing) errors could result in a maximum perturbation in the aperture center of 1 pixel, $\sim 2^{\prime \prime}$, but the change in galaxy contamination that would result is only $\leq 1 \%$. These tests indicate that changes in the seeing or inaccurate guiding or centering of the aperture can produce changes at the $1 \%$ level, but this is not sufficient to modulate the continua by $5 \%$. This increases our confidence in the reality of the wavelengthdependent lags observed.

To quantify and assess the significance of any wavelength-dependent lags, we first validate the estimated error bars of the lag determinations, represented in Figure 6. The first of two methods we considered is the rms method, as described by Wanders et al. (1997). Assuming the lag measurements of each wavelength bin are independent, we estimate $\sigma_{\mathrm{rms}}$ as the root mean square about the mean lag over $N$ wavelength bins. The three continuum regions considered were 5200-5320 $\AA$, 7000-7120 $\AA$, and 7360-7480 $\AA$. Table 4 details the results of the analysis. Column (1) is the central wavelength of the $N$ wavelength bins, column (2) is the number of bins, column (3) is the average lag over the $N$ bins, and column (4) is $\sigma_{\text {rms }}$. We note that $\sigma_{\mathrm{rms}}$ should be an upper limit, since it assumes a constant lag within each continuum region. We conclude that the error in the lag determinations per bin is 0.12 days. The second method was a "quasi-bootstrap" method, based on the bootstrap method (Press et al. 1992). Given a light curve with $N$ data points, we create 1000 synthetic light curves by selecting $N$ points from the original light curve at random and with replacement. Repeating the cross-correlation analysis for each of the 1000 synthetic data sets gives a probability distribution describing the uncertainty in the measured time delay, $\sigma_{\mathrm{BS}}$. Monte Carlo tests on Gaussian random numbers indicated that this procedure gives a distribution that is too narrow by a factor $[(N-1) / N]^{1 / 2}$. This correction factor is applied, but is very close to 1 for large $N$, within $\sim 1 \%$ for the sample size of our light curves. Using the ICCF algorithm to calculate the CCFs, the 4865 and $6962 \AA$ continua were found to lag the $1315 \AA$ light curve by $1.0 \pm 0.3$ and $1.5 \pm 0.7$ days, respectively. Note that in each synthetic data set some data points are omitted, others are duplicated. This is intended to modulate the weight given to each data point. However, because the ICCF algorithm simply interpolates the light curves the duplicated data points are also effectively removed. For this reason the quasi-bootstrap errors may be giving us conservative estimates of the error in the centroid determination. Indeed when these error bars are plotted in Figure 6, they are clearly larger than the point-to-point scatter in the time delays at different wavelengths. We note the rms error estimates may be underestimated due to some unknown correlation in the error estimates at different wavelengths. 


\section{DISCUSSION}

\subsection{Evidence of an Accretion Disk?}

The 1-2 day time delay between the UV and optical continuum variations is an intriguing result. Are we observing an extended reprocessing region? Could this be the signature of an accretion disk in this system, with harder photons from the center of the disk reprocessed into softer ones at radii farther out (Rokaki et al. 1992)? As mentioned in $\S 1$, previous campaigns indicated that continuum regions in NGC 5548 vary synchronously to within about \pm 1 day.

The observation of wavelength-dependent time delays offers a new opportunity to test the accretion disk model of an AGN. A steady state accretion disk has a temperature distribution (ignoring effects near the center) given by

$$
T^{4}=\frac{3 G M \dot{M}}{8 \pi \sigma R^{3}},
$$

where $T$ is the effective temperature, $R$ the radius from disk center, $M$ the mass of the central object, and $\dot{M}$ the accretion rate of the system. Continuum radiative reprocessing at radius $R$ incurs a mean time delay, $\tau=R / c$, which is independent of the disk inclination. The reprocessed light elevates the photospheric temperature of the disk, and if it resembles blackbody emission, it will peak at a wavelength $\lambda=h c / k T_{*} X$, where $X$ is of order unity and $T_{*}$ is the temperature of the disk due to viscous processes (eq. [4]) and heating of the disk photosphere through irradiation. We assume that departures from the viscous temperature and the $T \propto R^{-3 / 4}$ temperature structure (defined by eq. [4]) due to reprocessing are negligible. Substituting $\tau$ for $R$ and $\lambda$ for $T$ in equation (4), we find that for an irradiated blackbody accretion disk one expects

$$
\tau=3.1 \text { days }\left(\frac{M \dot{M}}{10^{6} M_{\odot}^{2} \mathrm{yr}^{-1}}\right)^{1 / 3}\left(\frac{\lambda}{10^{4} \AA}\right)^{4 / 3} .
$$

We have set $X=5.97$, i.e., where the $\partial B_{\lambda} / \partial T$ distribution peaks, to reflect the fact that we are interested in the sensitivity of the surface brightness distribution to temperature changes. Detailed modeling will be required to justify the use of this value of $X$.

Having established error bars, we now consider the significance of the wavelength-dependent lags by fitting a number of simple models to $\tau_{(\lambda)}$. We employ wavelength bins (Fig. 6) within 5200-5880 A, 6080-6520 A, and 7000$7760 \AA$, thereby excluding regions of dominant line emission. We include the data point at $1315 \AA$, where the lag is $\tau=0.00 \pm 0.08$ days. The results of our model fitting are summarized in Table 5. Column (1) details the model fitted to the data, column (2) details which lag uncertainty esti-

TABLE 5

Model FitTING to $\tau$ VERSUS $\lambda$ ReLATIONSHIP

\begin{tabular}{clccc}
\hline \hline $\begin{array}{c}\text { Model } \\
(1)\end{array}$ & \multicolumn{1}{c}{$\begin{array}{c}a_{1} \\
(2)\end{array}$} & $\begin{array}{c}\chi_{v}^{2} \\
(3)\end{array}$ & $\begin{array}{c}\text { (days) } \\
(4)\end{array}$ & $\begin{array}{c}a_{2} \\
(5)\end{array}$ \\
\hline$\tau=a_{1} \ldots \ldots \ldots \ldots \ldots \ldots$. & BS & 3.03 & $0.65 \pm 0.10$ & $\ldots$ \\
$\tau=a_{1}\left(\lambda_{*}^{4 / 3}-\lambda_{0}^{4 / 3}\right) \ldots \ldots$. & rms & 9.80 & $1.35 \pm 0.10$ & $\ldots$ \\
$\tau=a_{1}\left(\lambda_{*}^{a_{2}}-\lambda_{0}^{a_{2}}\right) \ldots \ldots \ldots$ & rms & 0.08 & $2.82 \pm 0.10$ & 1.33 \\
& BS & 0.08 & $2.81 \pm 0.03$ & 1.33 \\
& rms & 2.47 & $2.77 \pm 0.19$ & $1.40 \pm 0.33$ \\
& & & \\
\hline
\end{tabular}

NotE.-Here $\lambda_{*}=\lambda / 10^{4} \AA$ and $\lambda_{0}=0.1315=1315 / 10^{4} \AA$. mates were used in the fitting procedure $(\sigma=\mathrm{BS}$ denotes quasi-bootstrap error estimates, $\sigma=\mathrm{rms}$ denotes $\sigma_{\mathrm{rms}}=$ 0.12 days as described earlier), column (3) is the reduced $\chi^{2}$ of the model fit, $\chi_{v}^{2}$, and columns (4) and (5) report the relevant fitted parameter values for each model.

Model 1 tests the null hypothesis that the continuum lag is constant over all wavelengths, i.e., $\tau=a_{1}$. Using $\sigma_{\text {rms }}$, model 1 gives a very poor fit to the data as indicated by the large value of $\chi_{v}^{2}=9.80$, and the null hypothesis may be ruled out with great confidence. A reasonably good fit to the data is indicated by $\chi_{v}^{2} \approx 1$, provided the error estimates are realistic. Using the larger error bars $\sigma_{\mathrm{BS}}$, model 1 is still a poor fit to the data, $\chi_{v}^{2}=3.03$. We conclude that a constant time delay is inconsistent with the data.

Model 2 fits the function $\tau=a_{1}\left(\lambda_{*}^{4 / 3}-\lambda_{0}^{4 / 3}\right)$, and tests the $\tau \propto \lambda^{4 / 3}$ prediction for an irradiated blackbody accretion disk. Here $\lambda_{*}=\lambda / 10^{4} \AA$ and $\lambda_{0}=0.1315=1315 / 10^{4} \AA$. Model 2 gives $\chi_{v}^{2}=0.08$ using $\sigma_{\mathrm{BS}}$ and $\chi_{v}^{2}=2.43 \mathrm{using} \sigma_{\mathrm{rms}}$. The low $\chi_{v}^{2}$ obtained using $\sigma_{\mathrm{BS}}$ indicates that these error bars may be too large. Figure 7 presents the results of fitting model 2 to the data. The top panel assumes $\sigma_{\text {rms }}$, and the bottom panel uses the $\sigma_{\mathrm{BS}}$. The best-fit line for model 2 is detailed as the solid line in each panel.
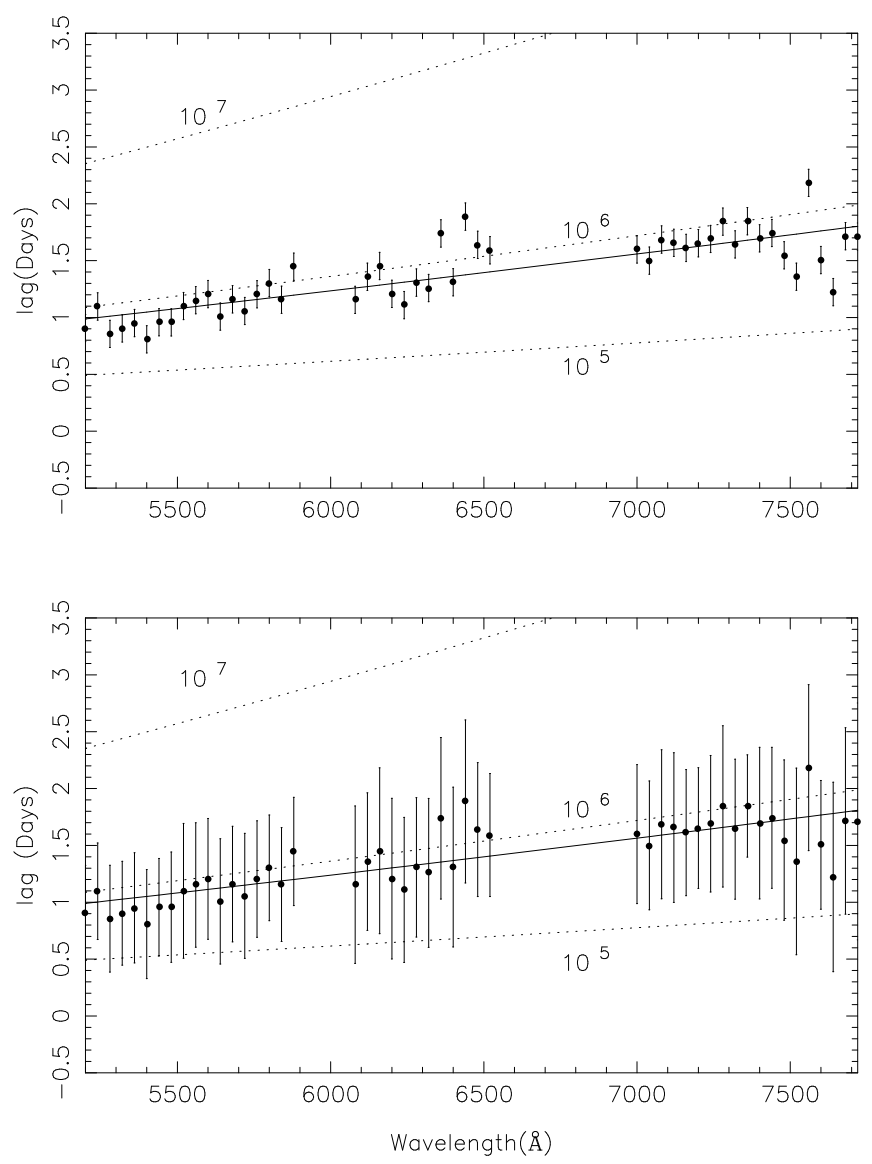

FIG. 7.- Irradiated blackbody accretion disk model $\tau=a_{1}\left(\lambda_{*}^{4 / 3}-\lambda_{0}^{4 / 3}\right)$ fitted to the wavelength-dependent time delays $\left(\lambda_{0}=0.1315\right.$ and $\lambda_{*}=$ $\lambda / 10^{4} \AA$ ). To exclude regions of dominant line emission only wavelength

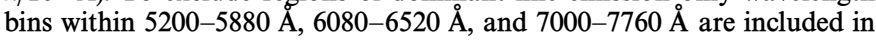
the fit. Top panel: Assumes constant lag uncertainties of 0.12 days. Solid line, best-fit line (model 2) to the data, $\chi_{v}^{2}=2.43$; dashed lines, $M \dot{M}=10^{7}$, $10^{6}$, and $10^{5} M_{\odot} \mathrm{yr}^{-1}$. Bottom panel: Identical to the top panel, but with lag uncertainties as estimated from the quasi-bootstrap procedure described in $\S 4.3$. The solid best-fit line has a $\chi_{v}^{2}=0.08$. We estimate $M \dot{M}=\left(0.7_{-0.4}^{+0.7}\right) \times 10^{6} M_{\odot} \mathrm{yr}^{-1}$. 
Model 3 fits the function $\tau=a_{1}\left(\lambda_{*}^{a_{2}}-\lambda_{0}^{a_{2}}\right)$, similar to model 2 except that the data is given more freedom to determine a best-fit wavelength dependence, $a_{2}$. The fits are similar to those obtained with model $2, \sigma_{\mathrm{BS}}$ yielding $\chi_{v}^{2}=$ 0.08 and $\sigma_{\text {rms }}$ giving a $\chi_{v}^{2}=2.47$. Model 3 tests the $T \propto R^{-3 / 4}$ law, which gives rise to $\tau \propto \lambda^{4 / 3}$.

Adopting $\sigma_{\mathrm{BS}}$, models 2 and 3 are both consistent within the quoted uncertainties and support the notion of a wavelength-dependent lag that is consistent with the prediction of a steady state irradiated blackbody accretion disk. Adopting the best-fit estimate for the parameter $a_{1}$ in model $3(\sigma=\mathrm{rms})$ we find that $a_{1}=2.8 \pm 0.7$ days, where the error estimate is the $68 \%$ confidence estimate corresponding to $\Delta \chi^{2} \pm 1$. This error estimate accounts for the correlation of the parameters $a_{1}$ and $a_{2}$.

\subsection{Mass and Accretion Rate of the Black Hole}

The best-fit parameter $a_{1}=2.8 \pm 0.7$ days in model 3 can be identified with the constant $3.1\left(M \dot{M} / 10^{6} M_{\odot}^{2} \mathrm{yr}^{-1}\right)^{1 / 3}$ days in equation (5) to estimate $M \dot{M}$. We estimate $M \dot{M}=$ $\left(7_{-0.4}^{+0.7}\right) \times 10^{6} M_{\odot}^{2} \mathrm{yr}^{-1}$. Lines of $M M=10^{5}, 10^{6}$, and $10^{7}$ $M_{\odot}^{2} \mathrm{yr}^{-1}$ are overplotted in each panel of Figure 7.

A crude estimate for the mass of the supposed black hole in NGC 7469 may be made. We calculate the "Keplerian mass" assuming an edge-on circular accretion disk with a velocity $V=\mathrm{FWHM} / 2$. For the "virial mass" we assume random motions of line emitting material in a spherical geometry, with a velocity $V=(\sqrt{3} / 2)$ FWHM (Netzer 1990). The mass is

$$
M=\frac{V^{2} \tau c}{G} .
$$

For the FWHM we use the velocity profile of the line from the rms spectrum in Figure 2, and for $\tau$ we use the crosscorrelation time delay, $\tau_{\text {cen }}$, with respect to the $1315 \AA$ light curve. The FWHM of the Balmer lines was measured by fitting a single Gaussian plus straight line model to the variable component of the line profile, Figure 2 . The fit to the $\mathrm{H} \beta$ line profile yielded a $\chi_{v}^{2}=0.7$. The fit to the $\mathrm{H} \alpha$ line profile yielded a poor $\chi_{v}^{2}=9.0$, due to imperfect wavelength calibration resulting in spurious peaks in the rms spectrum near each of the narrow lines. We reflect this fact in the error estimate for the FWHM measurement of this line. We note that multiple narrow and broad Gaussian component fits to the Balmer lines of the mean spectrum, Figure 2, yield estimates for the FWHMs of the lines to be $\sim 30 \%-40 \%$ larger than those estimated by fitting to the variable component of the lines. Table 6 summarizes the rough mass estimates. Column (1) is the emission-line feature considered, column (2) is the virial velocity $V$ of the emission line as measured from the rms spectrum in Figure 2, column (3) is the Keplerian velocity of the emission line as measured from the rms spectrum in Figure 2, column (4) is the time delay of the emission feature from the CCF (Fig. 5), column 5 is the virial mass estimate, and column (6) is the Keplerian mass of the central source.

The different mass estimates for the lines are internally consistent to within the quoted uncertainties. A weighted average of the mass estimates yields virial and Keplerian masses of $(8.3 \pm 2.4) \times 10^{6} M_{\odot}$ and $(2.7 \pm 0.8) \times 10^{6} M_{\odot}$, respectively. These two types of mass estimate indicate the large systematic uncertainty, depending on the unknown geometry and kinematics of the line emitting gas. Koratkar \& Gaskell (1991) derived the mass of the central object in 10 AGNs in a consistent manner. The central sources were found to have masses of about $10^{7}-10^{8} M_{\odot}$. Their mass estimates utilized the FWHM as measured from a mean spectrum compared to our measurement of the FWHM from an rms spectrum.

Combining the Keplerian mass estimate with the estimate of $M \dot{M}$ we derive an estimate for the accretion rate of the system, $\dot{M}=(0.3 \pm 0.2) M_{\odot} \mathrm{yr}^{-1}$. Assuming an accretion efficiency $\eta=0.1$, the bolometric luminosity of NGC 7469 would then be $\eta \dot{M} c^{2}=(1.7 \pm 1.1) \times 10^{45}$ ergs s $^{-1}$. The observed X-ray luminosity in the $0.5-4.5 \mathrm{keV}$ region is $10^{43.4} \mathrm{ergs} \mathrm{s}^{-1}$ (Green, Anderson, \& Ward 1992). We emphasize that the values for $M \dot{M}, M, \dot{M}$ and the bolometric luminosity are calculated under strict assumptions, as detailed in the text, that require extensive investigation.

\section{SUMMARY}

An intensive spectrophotometric optical monitoring campaign on the Seyfert 1 galaxy NGC 7469 was undertaken during 1996 June-July. The main results are summarized as follows:

1. The $\mathrm{H} \beta$ and $\mathrm{H} \alpha$ emission lines respond to the $1315 \AA$ continuum variations with a time delay of $\sim 5-6$ days. We consider this to be identification of variable Balmer line gas at a distance of $\sim 5-6$ light days from the central source.

2. By combining the emission-line lags and the widths of the variable component of the emission lines, we estimate the mass of the central source to be $M \sim 10^{6}-10^{7} M_{\odot}$.

3. Significant wavelength-dependent time delays are detected in the continuum. Variations at optical wavelengths lag behind those at $1315 \AA$. Cross correlation of the light curves shows the delay increases from $\sim 1$ day at 4800 $\AA$ to $\sim 2$ days at $7500 \AA$. We interpret this as evidence for a stratified continuum reprocessing region close to the central source, possibly that of an accretion disk. With this interpretation the delays are consistent with the predicted $\tau \propto$ $(M \dot{M})^{1 / 3} \lambda^{4 / 3}$ relationship for an irradiated blackbody accretion disk with temperature profile $T \propto R^{-3 / 4}$ and suggest $M \dot{M}=\left(0.7_{-0.4}^{+0.7}\right) \times 10^{6} M_{\odot}^{2} \mathrm{yr}^{-1}$.

TABLE 6

VIRIAL AND KePLERIAN MASS EstimateS

\begin{tabular}{cccccc}
\hline \hline $\begin{array}{c}\text { Emission Line } \\
(1)\end{array}$ & $\begin{array}{c}V_{\text {virial }} \\
\left(\mathrm{km} \mathrm{s}^{-1}\right) \\
(2)\end{array}$ & $\begin{array}{c}V_{\text {Keplerian }} \\
\left(\mathrm{km} \mathrm{s}^{-1}\right) \\
(3)\end{array}$ & $\begin{array}{c}R \\
(\text { light days }) \\
(4)\end{array}$ & $\begin{array}{c}M_{\text {virial }} \\
\left(10^{6} M_{\odot}\right) \\
(5)\end{array}$ & $\begin{array}{c}M_{\text {Keplerian }} \\
\left(10^{6} M_{\odot}\right) \\
(6)\end{array}$ \\
\hline $\mathrm{H} \alpha \ldots \ldots \ldots \ldots$. & $2343 \pm 300$ & $1353 \pm 300$ & $5.6 \pm 1.3$ & $6.0 \pm 4.5$ & $2.0 \pm 1.4$ \\
$\mathrm{H} \beta \ldots \ldots \ldots \ldots$ & $2934 \pm 152$ & $1694 \pm 152$ & $5.4 \pm 0.8$ & $9.1 \pm 2.8$ & $3.0 \pm 0.9$ \\
\hline
\end{tabular}


More detailed modeling and analysis of the results remains to be done to investigate these wavelengthdependent time delays and test the applicability of the disk hypothesis. Concurrently, we believe the potential implications of these observations require immediate observational verification. Principally, this is possible with a high signalto-noise ratio, photometric observations of comparable temporal resolution.
S. C. would like to thank the Wise Observatory staff for their endless support and advice throughout the monitoring campaign and PPARC for travel assistance and a studentship at St. Andrews. This research is also supported by a special grant from the Israel Science Foundation. We are grateful to the NSF for support of the OSU observations through grant AST 94-20080.

\section{REFERENCES}

Alexander, T. 1998, in Astronomical Time Series, ed. E. Leibowitz, D. Maoz, \& A. Sternberg (Dordrecht: Kluwer)

Alloin, D., et al. 1995, A\&A, 293, 293

Clavel, J., et al. 1991, ApJ, 366, 64

Crenshaw, D. W., et al. 1996, ApJ, 470, 322

Dietrich, M., et al. 1993, ApJ, 408, 416 .1998, ApJ, submitted

Edelson, R. A., et al. 1996, ApJ, 470, 364

Gaskell, C. M., \& Peterson, B. 1987, ApJS, 65, 1

Gaskell, C. M., \& Sparke, L. S. 1986, ApJ, 305,175

Green, P. J., Anderson, S. F., \& Ward, M. J. 1992, MNRAS, 254, 30

Kaspi, S., Ibbetson, P. A., Mashal, E., \& Brosch, N. 1996, Wise Obs. Tech. Rep., No. 6

Koratkar, A. P., \& Gaskell, C. M. 1991, ApJ, 370, L61

Korista, K., et al. 1995, ApJS, 97, 285

Kriss, G. A., et al. 1998, in preparation

Krolik, J. H., Horne, K., Kallman, T. R., Malkan, M. A., Edelson, R. A., \& Kriss, G. A. 1991, ApJ, 371, 541

Lang, K. R. 1980, Astrophysical Formulae (Berlin: Springer), 560

Leighly, K. M., O'Brien, P. T., Edelson, R., George, I. M., Malkan, M. A., Matsuoka, M., Mushotzky, R. F., \& Peterson, B. M. 1997, ApJ, 483, 767 Malkan, M., \& Filippenko, A. 1983, ApJ, 275, 477

Maoz, D., et al. 1990, ApJ, 351, 75 .1991, ApJ, 367, 493 .1993, ApJ, 404, 576

Marshall, H. L., et al. 1997, ApJ, 479, 222
Nandra, K., et al. 1998, in preparation

Netzer, H. 1990, in Active Galactic Nuclei, ed. T. J. L. Courvoisier \& M. Mayor (Berlin: Springer)

O'Brien, P. T., et al. 1998, ApJ, submitted

Penston, M. V., Penston, M. J., \& Sandage, A. 1971, PASP, 83, 783

Peterson, B. M. 1993, PASP, 105, 207

Peterson, B. M., et al. 1991, ApJ, 368, 119 .1992, ApJ, 392, 470

Peterson, B. M., Pogge, R. W., Wanders, I., Smith, S. M., \& Romanishin, W. 1995, PASP, 107,579

Press, W., et al. 1992, Numerical Recipes (Cambridge: Cambridge Univ. Press)

Reichert, G. A., et al. 1994, ApJ, 425, 582

Rodríguez-Pascual, P. M., et al. 1997, ApJS, 110, 9

Rokaki, E., et al. 1992, A\&A, 261, 41

Romanishin, W., et al. 1995, ApJ, 455, 516

Salamanca, I., Alloin, D., \& Pelat, D. 1995, A\&AS, 111, 283

Santos-Lleó, M., et al. 1997, ApJS, 112, 271

Stirpe, G., et al. 1994, ApJ, 425, 609

van Groningen, E., \& Wanders, I. 1992, PASP, 104, 700

Wade, R., \& Horne, K. 1988, ApJ, 324, 411

Wanders, I., et al. 1997, ApJS, 113, 69

Warwick, R. S., et al. 1996, ApJ, 470, 349

Welsh, W., et al. 1998, in preparation

White, R. J., \& Peterson, B. M. 1994, PASP, 106, 879 